\title{
NONEXISTENCE OF SOME COMPLETE STABLE MINIMAL SUBMANIFOLDS
}

\author{
By MAKOTO SAKaKI
}

\section{0 . Introduction.}

Simons [20] proved that there is no closed stable minimal submanifold in the unit sphere, and that there is no closed stable minimal hypersurface in any Riemannian manifold with positive Ricci curvature. Complete stable minimal surfaces in 3-dimensional Riemannian manifolds with nonnegative scalar curvature were discussed by Fischer-Colbrie and Schoen [5], and complete stable minimal hypersurfaces in the Euclidean space were discussed by do Carmo and Peng [3]. In Section 2 of this paper we deal with the nonexistence problem for some complete stable minimal submanifolds in the unit sphere and some complete stable minimal hypersurfaces in a Riemannian manifold with nonnegative Ricci curvature. In Section 3 we consider an application of the nonexistence argument to the free boundary problem of minimal surfaces.

The examples of complete non-compact minimal surfaces in the 3 and 5-dimensional unit sphere are obtained by use of the method in [9]. In [6] and [14] uncountably many examples of complete non-compact minimal hypersurfaces in the unit sphere are constructed.

In this paper all manifolds are smooth, connected, and have dimensions not less than 2. We shall use the same $\langle$,$\rangle to denote the inner products on fibers$ of vector bundles of Riemannian manifolds. We denote by $\lambda_{1}(M)$ the greatest lower bound of the spectrum of the Laplacian of a Riemannian manifold $M$.

The author would like to express his hearty thanks to Prof. S. Tanno for his constant encouragement and advice, and to the referee for his useful comments.

\section{Preliminaries.}

Let $f: M \rightarrow S^{n}$ be a minimal immersion of an $m$-dimensional Riemannian manifold $M$ into the $n$-dimensional unit sphere $S^{n}$ in the $(n+1)$-dimensional Euclidean space $R^{n+1}$. We denote by $\nabla$ the Riemannian connection of $M$. Let $\phi$ be any function on $M$ with compact support such that $\phi^{2}$ and $|\nabla \phi|^{2}$ are integrable on $M$ and $\phi=0$ on the boundary of $M$. Let $\left\{v_{1}, \cdots, v_{n+1}\right\}$ be parallel

Received September 7, 1987 
vector fields in $R^{n+1}$ which are orthonormal at each point. We consider the second variation $I\left(\psi v_{j}^{\perp}, \phi v_{j}^{\frac{1}{j}}\right)$ of the volume functional of $M$ for the variational vector field $\phi v_{j}^{\perp}$, where we denote by ()$^{\perp}$ the projection to normal space of $M$ in $S^{n}$.

PROPOSITION 1.1. Under the same notation as above.

$$
\sum_{j=1}^{n+1} I\left(\phi v_{\jmath}^{\perp}, \phi v_{j}^{\perp}\right)=(n-m) \int_{M}\left(|\nabla \phi|^{2}-m \psi^{2}\right) d M
$$

Proof. We denote by ()$^{T}$ the projection to the tangent space of $M$. Let $\partial$ be the flat connection of $R^{n+1}$. Then by the second variational formula for minimal submanifolds

$$
\begin{aligned}
I\left(\psi v_{j}^{\perp}, \phi v_{j}^{\perp}\right)= & \int_{M}\left\{\left|\left(\partial\left(\phi v_{j}^{\perp}\right)\right)^{\perp}\right|^{2}-\left|\left(\partial\left(\phi v_{j}^{1}\right)\right)^{T}\right|^{2}-m \psi^{2}\left|v_{j}^{\perp}\right|^{2}\right\} d M \\
=\int_{M} & {\left[|\nabla \phi|^{2}\left|v_{j}^{\perp}\right|^{2}+\frac{1}{2}\left\langle\nabla \psi^{2}, \nabla\left|v_{j}^{1}\right|^{2}\right\rangle\right.} \\
& \left.\quad+\psi^{2}\left\{\left|\left(\partial v_{j}^{\perp}\right)^{\perp}\right|^{2}-\left|\left(\partial v_{j}^{\perp}\right)^{T}\right|^{2}-m\left|v_{j}^{\perp}\right|^{2}\right\}\right] d M .
\end{aligned}
$$

As the ambient space is $S^{n}, v_{j}=v_{j}^{T}+v_{j}^{1}+\left\langle v_{j}, f(p)\right\rangle f(p)$ at each $p \in M$. Take the differentiation of this equation on $M$ and project to the normal space of $M$ in $S^{n}$. Then $0=\left(\partial v_{j}^{T}\right)^{\perp}+\left(\partial v_{j}^{\perp}\right)^{\perp}$. Thus we calculate

$$
\begin{aligned}
\sum_{j=1}^{n+1}\left|\left(\partial v_{\jmath}^{\perp}\right)^{\perp}\right|^{2} & =\sum_{j=1}^{n+1}\left|\left(\partial v_{j}^{T}\right)^{\perp}\right|^{2}=\sum_{j, k=1}^{n+1}\left\langle\partial v_{j}^{T}, v_{k}^{\perp}\right\rangle^{2} \\
& =\sum_{j, k=1}^{n+1}\left\langle v_{j}^{T}, \partial v_{k}^{\perp}\right\rangle^{2}=\sum_{k=1}^{n+1}\left|\left(\partial v_{k}^{\perp}\right)^{T}\right|^{2} .
\end{aligned}
$$

The proposition is shown by (1) and (2).

Q.E.D.

COROLlARY 1.2. Let $M$ be an m-dimensional minimal submanifold in the unit sphere, and assume that $\lambda_{1}(M)<m$. Then $M$ is unstable.

\section{Nonexistence theorems.}

THEOREM 2.1. In the unit sphere there is no m-dimensional complete stable minimal submanifold with Ricci curvature greater than $-4 m /(m-1)$.

Proof. Assume that there is an $m$-dimensional complete stable minimal submanifold $M$ with Ricci curvature greater than $-4 m /(m-1)$ in the unit sphere. From [20] we may assume that $M$ is non-compact. If the volume of $M$ is finite, then $\lambda_{1}(M)=0$, which implies the instability of $M$ by Corollary 1.2. So we may also assume that the volume of $M$ is infinite. Define a family of subsets $B(r)$ of $M$ by 


$$
B(r)=\{p \in M ; \rho(p) \leqq r\}
$$

where $\rho(p)$ is the geodesic distance on $M$ from $p$ to a fixed point on $M$. Let $V(r)$ be the volume of $B(r)$. The exponential growth $\mu$ of $M$ is defined to be $\lim _{r \rightarrow \infty} \sup (\log V(r)) / r$. As the Ricci curvature of $M$ is greater than $-4 m /(m-1)$, it follows from the comparison theorem [1, p. 253] that $\mu<2 m^{1 / 2}$. As $M$ is complete non-compact and the volume of $M$ is infinite, we find by Theorem 1 of [2] that $\lambda_{1}(M)<m$, which implies the instability of $M$ by Corollary 1.2. This contradiction completes the proof.

Q.E.D.

THEOREM 2.2. Let $M$ be an m-dimensional complete Riemannian manifold, and assume that the scalar curvature $S$ of $M$ satisfies

$$
\int_{M}(m(m-1)-S) d M<\infty \text { and } \int_{M}\left(\frac{m(m-1)\left(m^{2}-5\right)}{m^{2}+m-3}-S\right)(m(m-1)-S) d M<0 .
$$

Then there is no stable minimal immersion of $M$ into the unit sphere.

Proof. Assume that there is a stable minimal immersion of $M$ into the unit sphere. Let $\nabla$ and $\phi$ be as in Section 1 . We denote by $A$ and $\Delta$ the second fundamental form and the Laplacian of $M$, respectively. Then it follows from Proposition 1.1 that

$$
\begin{aligned}
0 & \leqq \int_{M}\left(|\nabla(\psi|A|)|^{2}-m(\psi|A|)^{2}\right) d M \\
& =\int_{M}\left(|\nabla \psi|^{2}|A|^{2}+\frac{1}{2}\left\langle\nabla \psi^{2}, \nabla|A|^{2}\right\rangle+\psi^{2}|\nabla| A||^{2}-m \psi^{2}|A|^{2}\right) d M \\
& =\int_{M}\left\{|\nabla \psi|^{2}|A|^{2}+\psi^{2}\left(-\frac{1}{2} \Delta|A|^{2}+|\nabla| A||^{2}-m|A|^{2}\right)\right\} d M
\end{aligned}
$$

In our previous paper [17] we have the following inequality:

$$
\begin{aligned}
-\frac{1}{2} \Delta|A|^{2}+|\nabla| A||^{2} & =-\langle\Delta A, A\rangle-|\nabla A|^{2}+|\nabla| A||^{2} \\
& \leqq-\langle\Delta A, A\rangle \leqq\left(2-\frac{2}{(m-1)(m+2)}\right)|A|^{4}-m|A|^{2} .
\end{aligned}
$$

Using (4), (5) and the Gauss equation $|A|^{2}=m(m-1)-S$, we have

$$
0 \leqq \int_{M}|\nabla \phi|^{2}(m(m-1)-S) d M+c_{1} \int_{M} \psi^{2}\left(\frac{m(m-1)\left(m^{2}-5\right)}{m^{2}+m-3}-S\right)(m(m-1)-S) d M
$$

for some $c_{1}>0$. We choose the function $\phi$ as follows :

$$
\phi(p)=\left\{\begin{array}{cl}
1 & \rho(p) \leqq R \\
2-\frac{\rho(p)}{R} & R \leqq \rho(p) \leqq 2 R \\
0 & \rho(p) \geqq 2 R
\end{array}\right.
$$


for $p \in M$, where $\rho(p)$ is defined as in (3) and $R$ is any positive number. Put this function into (6). Then using the intermediate-value theorem and the fact that $|\nabla \rho| \leqq 1$ almost everywhere on $M$, we have

$$
0 \leqq R^{-2} \int_{M}(m(m-1)-S) d M+c_{1} \int_{B(r)}\left(\frac{m(m-1)\left(m^{2}-5\right)}{m^{2}+m-3}-S\right)(m(m-1)-S) d M
$$

for some $r \in[R, 2 R]$, where $B(r)$ is defined as (3). Note that this inequality is true even if $M$ is compact. Because of the hypothesis, we have a contradiction by choosing $R$ sufficiently large in (8).

Q.E.D.

Remark. Let $M$ be an $m$-dimensional minimal submanifold in $S^{n}$, and let $\Delta$ denote the Laplacian of $M$. In [20] it is shown that the second fundamental form $A$ of $M$ satisfies $-\langle\Delta A, A\rangle \leqq\left\{2-(n-m)^{-1}\right\}|A|^{4}-m|A|^{2}$, which is better than (5) if $n \leqq(m-1)(m+4) / 2$. If we use it in the above proof instead of (5), we may have another result. And if we use $|A|^{\alpha}(\alpha \geqq 1)$ instead of $|A|$, we may have the other result.

Next we use the method in [5] and discuss the nonexistence for a complete stable minimal submanifold conformally equivalent to a bounded open domain in the Euclidean space or to a complete Riemannian manifold with nonnegative Ricci curvature. Note that a complete non-compact minimal surface in a Riemannian manifold has the universal covering space which is conformally equivalent to the open unit disk on $R^{2}$ or to $R^{2}$.

THEOREM 2.3. Let $M$ be an m-dimensional complete Riemannian manifold conformally equivalent to a bounded open domain in $R^{m}$.

(i) If the scalar curvature $S$ of $M$ satisfies $S \geqq-4(m-1)$, then there is no stable minimal immersion of $M$ into the unit sphere.

(ii) There is no stable minimal immersion of $M$ into any $(m+1)$-dimensional Riemannian manifold $N$ with nonnegative sectional curvature.

Proof. We may assume that the metric of $M$ is represented as

$$
d s^{2}=g\left(d x_{1}^{2}+\cdots+d x_{m}^{2}\right)
$$

by the coordinate system $\left(x_{1}, \cdots, x_{m}\right)$ over $M$ induced from $R^{m}$. Let $\nabla, \Delta$ and $\phi$ be as in the proof of Theorem 2.2.

First we prove (i). Assume that there is a stable minimal immersion of $M$ into the unit sphere. Then we calculate as in (4)

$$
\begin{aligned}
0 & \leqq \int_{M}\left(\left|\nabla\left(\psi g^{-m / 4}\right)\right|^{2}-m\left(\phi g^{-m / 4}\right)^{2}\right) d M \\
& =\int_{M}\left(|\nabla \psi|^{2} g^{-m / 2}-\psi^{2} g^{-m / 4} \Delta g^{-m / 4}-m \psi^{2} g^{-m / 2}\right) d M .
\end{aligned}
$$


By the computation we have

$$
\Delta g^{-m / 4}=\frac{m(m+4)}{16} g^{(-m / 4)-2}|\nabla g|^{2}-\frac{m}{4} g^{(-m / 4)-1} \Delta g
$$

and

$$
S=(m-1)\left(-g^{-1} \Delta g+\frac{m+2}{4} g^{-2}|\nabla g|^{2}\right) .
$$

From (10), (11) and (12) we have

$$
\begin{aligned}
0 & \leqq \int_{M}\left(|\nabla \phi|^{2} g^{-m / 2}-\frac{m}{8} \psi^{2} g^{(-m / 2)-2}|\nabla g|^{2}-\left(\frac{m S}{4(m-1)}+m\right) \psi^{2} g^{-m / 2}\right) d M \\
& \leqq \int_{M}|\nabla \psi|^{2} d x_{1} \cdots d x_{m}-\frac{m}{8} \int_{M} \phi^{2} g^{(-m / 2)-2}|\nabla g|^{2} d M
\end{aligned}
$$

under our hypothesis. Choose the function $\psi$ as (7) and put it into (13). Then

$$
0 \leqq R^{-2} \int_{M} d x_{1} \cdots d x_{m}-\frac{m}{8} \int_{B(R)} g^{(-m / 2)-2}|\nabla g|^{2} d M,
$$

where $B(R)$ is defined as (3). If $g$ is constant, then $M$ cannot be complete under our condition. Therefore $|\nabla g|$ is not identically zero on $M$. The hypothesis says that $\int_{M} d x_{1} \cdots d x_{m}$ is bounded from above. So we have a contradiction by choosing $R$ sufficiently large in (14), which completes the proof of (i).

Next we prove (ii). Assume that there is a stable minimal immersion of $M$ into $N$. We denote by $A$ and $R_{N}$ the second fundamental form of $M$ and the Ricci tensor of $N$, respectively. Let $\nu$ be the unit normal vector field to $M$. Then by the second variational formula for minimal submanifolds

$$
\begin{gathered}
0 \leqq \int_{M}\left\{\left|\nabla\left(\phi g^{-m / 4}\right)\right|^{2}-\left(\phi g^{-m / 4}\right)^{2}\left(|A|^{2}+R_{N}(\nu, \nu)\right)\right\} d M \\
\leqq \int_{M}\left\{|\nabla \phi|^{2} g^{-m / 2}-\frac{m}{8} \psi^{2} g^{(-m / 2)-2}|\nabla g|^{2}\right. \\
\left.\quad-\left(\frac{m S}{4(m-1)}+|A|^{2}+R_{N}(\nu, \nu)\right) \psi^{2} g^{-m / 2}\right\} d M .
\end{gathered}
$$

By use of the Gauss equation and the hypothesis, we can see that $(m S / 4(m-1))$ $+|A|^{2}+R_{N}(\nu, \nu)$ is nonnegative on $M$. The rest of the proof is identical to that of (i).

Q.E. D.

Using the computation in the above proof, we find the following fact.

Proposition 2.4. Let $M$ be an m-dimensional complete Riemannian manifold conformally equivalent to a bounded open domain in $R^{m}$. Then the scalar curvature of $M$ is negative at some point. 
Proof. Assume that the scalar curvature of $M$ is nonnegative at each point. Then using the same notation and computation as in the proof of Theorem 2.3, we have

$$
0 \leqq \int_{M}\left|\nabla\left(\psi g^{-m / 4}\right)\right|^{2} d M \leqq \int_{M}\left(|\nabla \psi|^{2} g^{-m / 2}-\frac{m}{8} \psi^{2} g^{(-m / 2)-2}|\nabla g|^{2}\right) d M .
$$

And we have a contradiction as above.

Q.E.D.

Remark. The $m$-dimensional hyperbolic space $H^{m}(a)$ with constant curvature $a<0$ is conformally equivalent to the open unit ball in $R^{m}$. So we find that Proposition 2.4 is strict by considering $H^{m}(a)$ for $a$ sufficiently close to 0 .

THEOREM 2.5. Let $M$ be an m-dimensional complete Riemannian manifold conformally equivalent to a complete Riemannian manifold $M^{\prime}$ with nonnegative Ricci curvatvre. Then there is no stable minimal immersion of $M$ into the unit sphere, and into any $(m+1)$-dimensional Riemannian manifold $N$ whose infimum of Ricci curvature is positive.

Proof. Let $\nabla$ be as in Section 1 . Let $\phi$ be any nonnegative function on $M$ with compact support such that $\phi^{m}$ and $|\nabla \psi|^{m}$ are integrable on $M$. Using the arithmetic-geometric mean inequality, we have

$$
\left|\nabla \psi^{m / 2}\right|^{2}=\frac{m^{2}}{4} \psi^{m-2}|\nabla \psi|^{2} \leqq \frac{m}{2} \varepsilon^{-m(m-2)}|\nabla \phi|^{m}+\frac{m(m-2)}{4} \varepsilon^{2 m} \psi^{m}
$$

for any positive number $\varepsilon$.

Assume that there is a stable minimal immersion of $M$ into the unit sphere. Then using Proposition 1.1 and (15) for a sufficiently small $\varepsilon$, we have

$$
0 \leqq \int_{M}\left(\left|\nabla \psi^{m / 2}\right|^{2}-m\left(\psi^{m / 2}\right)^{2}\right) d M \leqq c_{2} \int_{M}|\nabla \psi|^{m} d M-c_{3} \int_{M} \psi^{m} d M
$$

for some $c_{2}, c_{3}>0$. We choose the function $\psi$ as (7) except $\rho(p)$ is defined to be the geodesic distance on $M^{\prime}$ from $p \in M$ to a fixed point on $M$. Put this function into (16). The first integral of the right hand side of (16) is an conformal invariance, so we may compute it on $M^{\prime}$. Therefore, we have for some $c_{4}>0$

$$
0 \leqq R^{-m} V^{\prime}(2 R)-c_{4} V(R)
$$

Here $V^{\prime}(r)$ is the volume of $B(r)$ measured on $M^{\prime}$ and $V(r)$ is the volume of $B(r)$ measured on $M$, where $B(r)$ is defined as (3) except $\rho(p)$. As $M^{\prime}$ has nonnegative Ricci curvature, it follows from the comparison theorem that $R^{-m} V^{\prime}(2 R)$ is bounded from above. Thus we find that the volume of $M$ is finite by choosing $R$ sufficiently large in (17). Then $\lambda_{1}(M)=0$, which implies the instability of $M$ by Corollary 1.2. This contradiction completes the proof. 
The second statement is shown similarly.

Q.E.D.

Let $K$ denote the Gaussian curvature of a 2-dimensional Riemannian manifold $M$, and set $C^{-}=\int_{M} \max \{-K, 0\} d M$.

COROLLARY 2.6. In the unit sphere there is no complete stable minimal surface with finite $C^{-}$.

Proof. Any complete non-compact 2-dimensional Riemannian manifold with finite $C^{-}$is parabolic (see [7]). Thus the assertion is proved by use of Theorem 2.5 .

Q.E. D.

\section{An application.}

Let $M$ be a hypersurface in a Riemannian manifold, and assume that the unit normal vector field to $M$ is given. The hypersurface $M$ is said to be $p$-convex and weakly $p$-convex if and only if the sum of any $p$ principal curvatures at each point with respect to the given normal vector is positive and nonnegative, respectively (see [19]).

In this section we use the method in [13] and show the following results:

THEOREM 3.1. Let $M$ be a closed hypersurface embedded in $R^{n}$. Let $X$ be the closure of the unbounded component of $R^{n}-M$, and let $Y$ be the closure of the complement of $X$ in $R^{n}$. Choose the unit normal vector field to $M$ toward $Y$.

(i) ([13]) If $M$ is (n-2)-convex, then $X$ is simply connected.

(ii) If $n \geqq 4$ and $M$ is 2-convex, then the map $i_{*}: \pi_{1}(M) \rightarrow \pi_{1}(Y)$ induced from the inclusion $i: M \rightarrow Y$ is an isomorphism.

THEOREM 3.2. Let $M$ be a closed hypersurface embedded in $S^{n}$. Let $X$ and $Y$ be the closures of two distinct components of $S^{n}-M$. Choose the unit normal vector field to $M$ toward $Y$.

(i) If $M$ is weakly (n-2)-convex, then $X$ is simply connected.

(ii) If $n \geqq 4$ and $M$ is 2-convex, then the map $i_{*}: \pi_{1}(M) \rightarrow \pi_{1}(Y)$ induced from the inclusion $i: M \rightarrow Y$ is an isomorphism.

We prove only the latter, because the proof of Theorem 3.1(ii) is similar to that of Theorem 3.2(ii). First we show two lemmas for the preparation.

If a map $f$ from a 2-dimensional manifold $L$ to a Riemannian manifold is critical for the area functional of $L$ and the points in the interior of $L$ at which $f$ is not an immersion are isolated, then $f$ is called a branched minimal immersion and the points at which $f$ is not an immersion are called the branch points of $f$.

Let $f: L \rightarrow S^{n}$ be a branched minimal immersion of a 2-dimensional manifold $L$ into $S^{n}$, and let $L^{\prime}$ denote the complement of the branch points on $L$. In 
the following we shall assume that $L$ admits the metric induced by $f$ from $S^{n}$, which is zero at the branch points. Let $j: S^{n} \rightarrow R^{n+1}$ be the inclusion. We denote by $G_{2}(n+1)$ the Grassmannian manifold of 2-dimensional planes through the origin in $R^{n+1}$. Then the Gauss map $\gamma: L^{\prime} \rightarrow G_{2}(n+1)$ of the isometric immersion $\left.j \circ f\right|_{L^{\prime}}: L^{\prime} \rightarrow R^{n+1}$ is defined on $L^{\prime}$.

LEMMA 3.3. Under the same notation as above, the map $\gamma: L^{\prime} \rightarrow G_{2}(n+1)$ extends smoothly at the branch points in the interior of $L$.

Proof. As $\left.f\right|_{L^{\prime}}$ is a minimal immersion, the mean curvature vector of $\left.j \circ f\right|_{L^{\prime}}$ is parallel on $L^{\prime}$ (see [4]). Thus it follows from [15] that $\gamma$ is harmonic on $L^{\prime}$. Therefore we can see our assertion by use of Theorem 3.6 of [16].

Q.E.D.

The next lemma is shown exactly as in [12]. Let $N$ be a compact Riemannian manifold with boundary $\partial N$ to which the inner unit normal vector field is given. Let $\Gamma$ be a Jordan curve on $\partial N$. We denote by $D$ the set $\left\{(x, y) \in R^{2} ; x^{2}+y^{2}<1\right\}$ with its closure $\bar{D}$. Let $\mathscr{F}$ be the family of all $L_{1}^{2}$-maps $f:(\bar{D}, \partial D) \rightarrow(N, \Gamma)$.

LEMMA 3.4. If $\partial N$ is 2-convex and $\mathscr{F}$ is not empty under the same notation as above, then there exists a weakly conformal harmonic map in $\mathbb{I}$ which has least area among $\mathscr{I}$ with respect to the metric induced from $N$.

Proof of Theorem 3.2. First we prove (i). Assume that $X$ is not simply connected. Let $\mathscr{F}_{0}$ be the family of all smooth maps $f:(\bar{D}, \partial D) \rightarrow\left(S^{n}, X\right)$ such that $\left.f\right|_{\partial D}$ represent nontrivial elements of $\pi_{1}(X)$. Let $M(\varepsilon)$ be the $\varepsilon$-neighborhood of $M$ in $S^{n}$ for $\varepsilon>0$. Then there exists a positive number $\varepsilon_{0}$ such that $M$ is a deformation retract of $M(\varepsilon)$ for each $\varepsilon \leqq \varepsilon_{0}$. Let $\mathscr{I}_{1}$ be the family of all smooth maps $f: D \rightarrow S^{n}$ such that:

(1) The total energy of $f$ is finite.

(2) Given $\varepsilon<\varepsilon_{0}$, there exists an $r<1$ such that $f(D-D(r)) \subset M(\varepsilon)$, where $D(r)=\left\{(x, y) \in D ; x^{2}+y^{2}<r^{2}\right\}$, and

(3) $\left.f\right|_{\partial D(r)}$ represents a nontrivial element of $\pi_{1}(X \cup M(\varepsilon))$.

Then it follows from the argument in [11] and [13] that there exists a weakly conformal harmonic map $f_{0}$ in $\mathscr{I}_{0}$ which has least area among $\mathscr{I}_{1}$ and $f_{0}$ maps the normal vector of $\partial D$ to a normal vector of $M$.

It is standard that we may regard $f_{0}: \bar{D} \rightarrow S^{n}$ as a branched minimal immersion. In the following we shall assume that $\bar{D}$ admits the metric induced by $f_{0}$ from $S^{n}$. Let $\left\{v_{1}, \cdots, v_{n+1}\right\}$ be defined as in Section 1. If $p \in \bar{D}$ is not a branch point, we define at $p$ the projection ()$^{T}$ to the tangent space of $\bar{D}$ and the projection ( $)^{\perp}$ to the normal space of $\bar{D}$ in $S^{n}$, which extend smoothly at the branch points on $D$ by Lemma 3.3. From the property of $f_{0}$, we may consider the variation $f_{t} \in \mathscr{T}_{1}$ of $f_{0}$ for the variational vector field $v_{j}^{\perp}$, and we may compute the second variation $I\left(v_{j}^{\perp}, v_{j}^{\perp}\right)$ of the area functional of $D$. Let $\partial$ be the 
flat connection of $R^{n+1}$, let $h$ be the second fundamental form of $M$ in $S^{n}$, and let $\nu$ be the unit normal vector field to $M$ toward $Y$. We denote by $d v$ and $d s$ the area element of $D$ and the line element of $\partial D$, respectively. Then from the same argument as in [13] and the computation in Section 1, we calculate

$$
\begin{aligned}
\sum_{j=1}^{n+1} I\left(v_{j}^{\perp}, v_{j}^{\perp}\right) & =\sum_{j=1}^{n+1}\left\{\int_{D}\left(\left|\left(\partial v_{j}^{\perp}\right)^{\perp}\right|^{2}-\left|\left(\partial v_{j}^{\perp}\right)^{T}\right|^{2}-2\left|v_{j}^{\perp}\right|^{2}\right) d v-\int_{\partial D}\left\langle h\left(v_{j}^{\perp}, v_{j}^{\perp}\right), \nu\right\rangle d s\right\} \\
& =-2(n-2) \operatorname{area}(D)-\int_{\partial D} \sum_{j=1}^{n+1}\left\langle h\left(v_{j}^{\perp}, v_{j}^{\perp}\right), \nu\right\rangle d s .
\end{aligned}
$$

Here we may assume that $v_{n-1}$ is tangent to $\partial D, v_{n}=\nu$, and $v_{n+1}=f_{0}(p)$ at each $p \in \partial D$ because the value of $\sum_{j=1}^{n+1}\left\langle h\left(v_{j}^{\perp}, v_{j}^{\perp}\right), \nu\right\rangle$ is independent of the choice of $\left\{v_{j}\right\}$. Then by the property of $f_{0}$, we have

$$
\sum_{j=1}^{n+1} I\left(v_{j}^{\perp}, v_{j}^{\perp}\right)=-2(n-2) \operatorname{area}(D)-\int_{\partial D} \sum_{j=1}^{n-2}\left\langle h\left(v_{j}, v_{j}\right), \nu\right\rangle d s .
$$

The hypothesis says that the right hand side of (18) is negative, which implies a contradiction.

Next we prove (ii). Assume that the map $i_{*}$ is not injective. Then $\pi_{1}(M) \neq 0$. Let $\mathscr{T}_{0}^{\prime}$ and $\mathscr{F}_{1}^{\prime}$ be defined as $\mathscr{I}_{0}$ and $\mathscr{T}_{1}$ in the proof of (i) respectively, except we replace $S^{n}$ by $Y$ and $X$ by $M$. Then using the argument in [11] and [13] with Lemma 3.4, we can see that there exists a weakly conformal harmonic map $f_{0}^{\prime}$ in $\mathscr{T}_{0}^{\prime}$ which has least area among $\mathscr{F}_{1}^{\prime}$ and $f_{0}^{\prime}$ maps the normal vector of $\partial D$ to a normal vector of $M$. Thus the same argument as in (i) yields a contradiction. The surjectivity of $i_{*}$ is shown by use of Theorem 1 of $[10]$.

Q.E.D.

Remark. It is announced in [19] that Theorem 3.1(i) is shown from the following theorem:

THEOREM 3.5 ([19]). If a compact Riemannian manifold $N$ has nonnegative sectional curvature and p-convex boundary $\partial N$ to which the inner unit normal vector field is given, then $N$ has the homotopy type of a $C W$-complex of dimension not greater than $p-1$.

We can see that the above statement is true also in the case where $N$ has positive sectional curvature and $\partial N$ is 'weakly $p$-convex, because $\partial N$ becomes $p$-convex by multiplying the metric on $N$ by a function $\varphi$ which is sufficiently close to 1 in $C^{2}$-norm and satisfies $d \varphi(\nu)<0$ on $\partial N$ where $\nu$ is the inner unit normal vector field to $\partial N$. Thus also Theorem $3.2(\mathrm{i})$ is shown from the above theorem. 


\section{REFERENCES}

[1] R. Bishop and R. CRittenden, Geometry of Manifolds, New York-London, Academic Press, 1964.

[2] R. Brooks, A relation between growth and the spectrum of the Laplacian, Math. Z., 178 (1981), 501-508.

[3] M. Do CARmo and C.K. Peng, Stable complete minimal hypersurfaces, Proceedings of the 1980 Beijing Symposium on Differential Geometry and Differential Equations, Science Press, Beijing, 1982, 1349-1358.

[4] J. Eells and L. Lemaire, Selected Topics in Harmonic Maps, C. B. M. S. Regional Conference Series, 50, Amer. Math. Soc., 1983.

[5] D. Fischer-Colbrie And R. Schoen, The structure of complete stable minimal surfaces in 3-manifolds of non-negative scalar curvature, Comm. Pure. Appl. Math., 33 (1980), 199-211.

[6] W. Y. Hsiang and H.B. Lawson, Minimal submanifolds of low cohomogeneity, J. Diff. Geom., 5 (1971), 1-38.

[7] A. Huber, On subharmonic functions and differential geometry in the large, Comment. Math. Helv., 32 (1957), 13-72.

[8] W. JAGER, Behavior of minimal surfaces with free boundaries, Comm. Pure. Appl. Math., 23 (1970), 803-818.

[9] H. B. LAwson, Complete minimal surfaces in $S^{3}$, Ann. of Math., 92 (1970), 335-374.

[10] H. B. LAwson, The unknottedness of minimal embeddengs, Invent. Math., 11 (1970), 183-187.

[11] W.H. MeEKs and S.T. YaU, Topology of three dimensional manifolds and the embedding problems in minimal surface theory, Ann. of Math., 112 (1980), 441-484.

[12] W.H. MeEks and S.T. YAU, The existence of embedded minimal surfaces and the problem of uniqueness, Math. Z., 179 (1982), 151-168.

[13] J.D. Moore And T. Schulte, Minimal disks and compact hypersurfaces in Euclidean space, Proc. Amer. Math. Soc., 94 (1985), 321-328.

[14] T. Otsuki, Minimal hypersurfaces in a Riemannian manifold of constant curvature, Amer. J. Math., 92 (1970), 145-173.

[15] E. A. Ruh AND J. Vilms, The tension field of the Gauss map, Trans. Amer. Math. Soc., 149 (1970), 569-573.

[16] J. SACKS AND K. UHLENBECK, The existence of minimal immersions of 2-spheres, Ann. of Math., 113 (1981), 1-24.

[17] M. SAKAKI, Remarks on the rigidity and stability of minimal submanifolds, preprint.

[18] R. Schoen AND K. Uhlenbeck, Regularity of minimizing harmonic maps into the sphere, Invent. Math., 78 (1984), 89-100.

[19] J.P. SHA, p-convex Riemannian manifolds, Invent. Math., 83 (1986), 437-447.

[20] J. Simons, Minimal varieties in riemannian manifolds, Ann. of Math., 88 (1968), 62-105.

Department of Mathematics

TOKYo Institute of TECHNOLOGY

Oh-okayama, Meguro-Ku, Tokyo 152

JAPAN 